\title{
Extracellular Vesicles Isolated from Human Induced Pluripotent Stem Cell-Derived Neurons Contain a Transcriptional Network
}

\author{
David A. Hicks ${ }^{1}$ (D) Alys C. Jones ${ }^{1,3} \cdot$ Nicola J. Corbett $^{1,4} \cdot$ Kate Fisher $^{1} \cdot$ Stuart M. Pickering-Brown ${ }^{1} \cdot$ Mark P. Ashe $^{2}$. \\ Nigel M. Hooper ${ }^{1}$
}

Received: 25 November 2019 / Revised: 23 March 2020 / Accepted: 27 March 2020 / Published online: 2 May 2020

(c) The Author(s) 2020

\begin{abstract}
Healthy brain function is mediated by several complementary signalling pathways, many of which are driven by extracellular vesicles (EVs). EVs are heterogeneous in both size and cargo and are constitutively released from cells into the extracellular milieu. They are subsequently trafficked to recipient cells, whereupon their entry can modify the cellular phenotype. Here, in order to further analyse the mRNA and protein cargo of neuronal EVs, we isolated EVs by size exclusion chromatography from human induced pluripotent stem cell (iPSC)-derived neurons. Electron microscopy and dynamic light scattering revealed that the isolated EVs had a diameter of 30-100 nm. Transcriptomic and proteomics analyses of the EVs and neurons identified key molecules enriched in the EVs involved in cell surface interaction (integrins and collagens), internalisation pathways (clathrin- and caveolin-dependent), downstream signalling pathways (phospholipases, integrin-linked kinase and MAPKs), and long-term impacts on cellular development and maintenance. Overall, we show that key signalling networks and mechanisms are enriched in EVs isolated from human iPSC-derived neurons.
\end{abstract}

Keywords Extracellular vesicles $\cdot$ Neurons $\cdot$ RNA seq $\cdot$ Proteomics $\cdot$ Cell signalling

Electronic supplementary material The online version of this article (https://doi.org/10.1007/s11064-020-03019-w) contains supplementary material, which is available to authorized users.

David A. Hicks

david.hicks-2@manchester.ac.uk

Nigel M. Hooper

nigel.hooper@manchester.ac.uk

1 Division of Neuroscience and Experimental Psychology, School of Biological Sciences, Faculty of Biology, Medicine and Health, Manchester Academic Health Science Centre, University of Manchester, Manchester M13 9PT, UK

2 Division of Molecular \& Cellular Function, School of Biological Sciences, Faculty of Biology, Medicine and Health, Manchester Academic Health Science Centre, University of Manchester, Manchester M13 9PT, UK

3 Present Address: Manchester University NHS Foundation Trust, Manchester, UK

4 Present Address: Discovery Research UK, MSD, London, UK

\section{Introduction}

Extracellular vesicles (EVs) represent a major contact-independent mechanism for intercellular communication in the brain [1]. EVs comprise a wide range of sizes, cargoes and subcellular origins [2, 3]. Small EVs, defined as having a diameter of approximately $50-150 \mathrm{~nm}$, include exosomes which are of endosomal origin [4, 5]. EVs are released from the cell and contain a heterogeneous population of macromolecules, including lipids, proteins and various classes of RNA [2]. The protein cargo can be transferred to recipient cells, for example being endocytosed by dendritic cells and the cargo presented by MHC class I molecules to $\mathrm{CD} 4^{+} \mathrm{T}$ cells [6]. The mRNA cargo can be translationally competent as demonstrated by microarray analysis in recipient cells [7] and translation of Arc protein in recipient cells [8], which may modulate synaptic function. In addition, EVs have been implicated as a conduit for the cell to cell transfer of several misfolded proteins in neurodegenerative diseases, which may be a mechanism for the spread of pathogenic protein conformers in neurodegeneration [9]. A range of cellular stresses, including hypoxia and hypoglycaemia have been shown to modify EV cargo, including mRNA [10-15]. 
The biogenesis of small EVs (sEVs) is a complex process, involving a multiplicity of accessory proteins and has been comprehensively reviewed $[2,3]$. In brief, as endosomes mature, they fill with intraluminal vesicles to form a multivesicular body (MVB) [16]. The biogenesis can be ESCRTdependent, or independent, driven by tetraspanins and syntenin [2]. Hence ESCRT components and accessories, such as Tsg101 or Alix and tetraspanins (usually CD9, CD63 and CD81) all represent common sEV markers [17], although cell surface tetraspanin localisation has been reported [18]. The contents of the MVB can be degraded by the lysosome or released into the extracellular milieu [16]. The mechanisms driving sEV secretion are not fully understood, but certain Rab proteins (e.g. Rab27), lipids (e.g. ceramide and sphingomyelin) and SNAREs have all been shown to have important roles in the process [19-22].

Despite the potential involvement of EVs in neuronal intercellular communication [23], there is limited information on the cargo within EVs secreted from neurons. In this study we have isolated EVs from human neurons derived from induced pluripotent stem cells (iPSCs). Transcriptomic, proteomics and bioinformatics analyses were then performed on the EVs to identify the molecules and pathways contained within the neuronal sEVs. We show that sEV-enriched mRNAs and proteins are significantly linked to processes involved in the development and maintenance of the nervous system, forming defined signalling networks.

\section{Material and Methods}

\section{Materials}

All chemicals were purchased from Fisher Scientific (Loughborough, Leicestershire, UK) unless otherwise stated.

\section{Methods}

\section{Cell Culture}

The iPSC line, OX1-19 (obtained from S. Cowley, University of Oxford) [24-26] was maintained on Matrigel (BD Biosciences, Wokingham, Berkshire, UK) in mTeSR1 medium (StemCell Technologies, Cambridge, UK) containing $50 \mathrm{U} / \mathrm{ml}$ penicillin and $50 \mu \mathrm{g} / \mathrm{ml}$ streptomycin (SigmaAldrich, Gillingham, Dorset, UK) in a humidified incubator at $37{ }^{\circ} \mathrm{C}$ in a $5 \% \mathrm{CO}_{2}, 95 \%$ air atmosphere. Pluripotency and successful cortical neuron differentiation were confirmed using immunofluorescence microscopy with appropriate markers (pluripotency: Sox2, SSEA4, Oct4 and Nanog; mature neuron: MAP2, $\beta$ III tubulin, Tbr1 and Sat2b). The iPSCs were differentiated to cortical neurons as described previously [27], using dual-SMAD inhibition by $1 \mu \mathrm{M}$ dorsomorphin and $10 \mu \mathrm{M} \mathrm{SB} 431452$ (Tocris, Bio-techne). Following successful differentiation, neural progenitor cells were re-plated on day 35 post-induction at 300,000 cells/ well onto poly-ornithine and laminin-coated (SigmaAldrich) 6-well polystyrene tissue culture plates (Greiner Bio One, Stonehouse, Glos, UK) and neurons cultured until day $75+$ post-induction with media changes every 2-3 days. Post-induction culture medium was 1:1 DMEM F12: neurobasal medium containing B27 and N2 supplements, $2 \mathrm{mM}$ L-glutamine, $100 \mu \mathrm{M}$ 2-mercaptoethanol, $25 \mu \mathrm{M}$ insulin, $100 \mathrm{U} / \mathrm{mL}$ penicillin, $100 \mu \mathrm{g} / \mathrm{mL}$ streptomycin (all Life Technologies) and $0.5 \%$ non-essential amino acids (SigmaAldrich) at $200 \mu \mathrm{l} . \mathrm{cm}^{-2}$ culture area. Minimal cell death was observed microscopically during the culture period. Separate inductions from iPSCs to neurons were regarded as biological replicates and $n=3$, unless otherwise stated.

\section{Immunocytochemistry}

iPSCs or iPSC-derived neurons were cultured as described above, then washed three times in PBS before fixation in $4 \%$ paraformaldehyde for $10 \mathrm{~min}$. Cells were washed in PBS, then permeabilised in $0.2 \%$ Triton X-100 in PBS for $5 \mathrm{~min}$. After further washing, cells were incubated in blocking buffer (10\% donkey serum in PBS) in PBS for $3 \mathrm{~h}$ at room temperature followed by incubation with primary antibody (overnight, $4{ }^{\circ} \mathrm{C}$ ). Cells were then washed, incubated with secondary antibody (conjugated to Alexa Fluor 488 (RRID: AB_2556542) or Alexa Fluor 568 (AB_25340); Life Technologies, Paisley, UK), followed by washing in PBS and mounting on slides using DAPI-containing mounting medium (Southern Biotech, Birmingham, AL, USA). Slides were visualised using the Evos FL (Thermo Fisher Scientific, Loughborough, UK). Primary antibodies used were for: Sox2 (AB_2341193), SSEA4 (AB_778073), Oct4 (AB_445175), Nanog (AB_446437), MAP2 (AB_297885), $\beta I I I$ tubulin (AB_444319), Tbr1 (AB_2200219) and Sat2b (AB_882455) (all Abcam, Cambridge, UK).

\section{EV Isolation}

Conditioned medium from $5 \times 10^{6}$ cells was harvested by pipetting from neuronal cultures (approximately $50 \mathrm{ml}$ total) and centrifuged in polypropylene tubes $(300 \times g$ for $10 \mathrm{~min}$, then $2000 \times g$ for $20 \mathrm{~min}$ at $4{ }^{\circ} \mathrm{C}$ with maximum brake). Medium was then filtered through a $0.22 \mu \mathrm{m}$ filter into a Vivaspin 20 (100 kDa MWCO) centrifugal concentrator, followed by centrifugation at $3900 \times g\left(4{ }^{\circ} \mathrm{C}\right.$, maximum brake $)$ to reduce the volume to $0.5 \mathrm{ml}$. The concentrated medium was then added to a $\mathrm{qEV}$ original column (Izon Sciences, Oxford, UK) and separated by size exclusion chromatography (SEC). The first six fractions represented the void volume, with vesicles eluted in filtered phosphate-buffered 
saline (PBS) in subsequent fractions of $0.5 \mathrm{ml}$. After EV isolation, RNA/ protein cargo was isolated immediately. SEC is considered an intermediate recovery, intermediate specificity technique. Separate inductions from iPSCs to neurons were regarded as biological replicates and $n=3$, unless otherwise stated.

\section{Electron Microscopy}

The vesicular fractions (fractions 7-9) were pooled and centrifuged at $100,000 \times g$ to pellet the EVss. The samples were fixed with $4 \%$ formaldehyde $+2.5 \%$ glutaraldehyde in $0.1 \mathrm{M}$ HEPES buffer ( $\mathrm{pH}$ 7.2). Samples were post-fixed with $1 \%$ osmium tetroxide $+1.5 \%$ potassium ferrocyanide in $0.1 \mathrm{M}$ cacodylate buffer $(\mathrm{pH} 7.2)$ for $1 \mathrm{~h}$, then in $1 \%$ uranyl acetate in water overnight. The samples were dehydrated in ethanol infiltrated with Low Viscosity resin (TAAB Laboratory and Microscopy, Aldermaston, Berks, UK) and polymerized for $24 \mathrm{~h}$ at $60{ }^{\circ} \mathrm{C}$. Sections were cut with a Reichert Ultracut ultramicrotome and observed with FEI Tecnai 12 Biotwin microscope at $100 \mathrm{kV}$ accelerating voltage. Images were taken with Gatan Orius SC1000 CCD camera. EV diameter was calculated using ImageJ (NIH, USA).

\section{Dynamic Light Scattering}

Unconcentrated fractions eluted from the $\mathrm{qEV}$ column were analysed for particle diameter using the Zetasizer Nano (Malvern Panalytical, Malvern, Worcestershire, UK). Three analyses were performed per sample.

\section{Cell Lysis}

Cells were washed twice in ice-cold PBS and harvested in PBS. Cells were pelleted at $3000 \times g$ for $5 \mathrm{~min}\left(4^{\circ} \mathrm{C}\right)$ and resuspended in $6 \times$ volume of lysis buffer (RIPA buffer: $50 \mathrm{mM}$ Tris-HCl (pH 8.0), $150 \mathrm{mM}$ sodium chloride, 1\% Igepal CA-630 (Sigma-Aldrich), 0.5\% sodium deoxycholate, 0.1\% SDS, $1 \mathrm{mM}$ sodium fluoride, $1 \mathrm{mM}$ sodium orthovanadate, and Complete Protease Inhibitor cocktail (Roche Diagnostics, Burgess Hill, West Sussex, UK)). Lysis was performed for $30 \mathrm{~min}$ on ice, followed by centrifugation at $3000 \times \mathrm{gs}$ for $30 \mathrm{~min}\left(4^{\circ} \mathrm{C}\right)$ to yield the RIPA-soluble fraction as the supernatant, which was used for immunoblotting.

\section{SDS-PAGE and Immunoblotting}

SEV fractions eluted from the qEV column were concentrated ten-fold with an Amicon 10 centrifugal concentrator and then boiled for $5 \mathrm{~min}$ in $5 \times$ SDS-PAGE sample buffer containing DTT (Jena Biosciences, Jena, Germany). Samples were separated by electrophoresis $120 \mathrm{~V}$ for $90 \mathrm{~min}$ on a polyacrylamide gel containing $10 \%$ acrylamide. After
SDS-PAGE, proteins were transferred to polyvinylidene fluoride (PVDF) membranes for $75 \mathrm{~min}$ at $125 \mathrm{~V}$ (Bio-Rad). The PVDF membranes were incubated for $2 \mathrm{~h}$ in blocking solution $(5 \%(\mathrm{w} / \mathrm{v})$ milk power, $2 \%(\mathrm{w} / \mathrm{v}) \mathrm{BSA}$ in TBS $+1 \%$ $(\mathrm{v} / \mathrm{v})$ Tween-20 (TBST)) and then incubated overnight in primary antibody $(5 \%(\mathrm{w} / \mathrm{v})$ milk powder in TBS). The PVDF membranes were washed $4 \times 10$ min with TBST before the addition of secondary antibody (HRP-conjugated anti-IgG; 5\% (w/v) milk powder in TBST, 1:5000 (Thermo Fisher Scientific)) for $1 \mathrm{~h}$, followed by $4 \times 10 \mathrm{~min}$ washes with TBST. Protein bands were visualized and, where appropriate, quantified, by chemiluminescence (Clarity Western ECL Blotting Substrate, Bio-Rad) using a G:BOX and GeneTools software (Syngene, Cambridge, UK). Alternatively, polyacrylamide gels were stained with Coomassie Blue (R-250 Brilliant Blue in $45 \%$ methanol, $45 \% \mathrm{H}_{2} \mathrm{O}$, $10 \%$ glacial acetic acid) for $30 \mathrm{~min}$ and destained for $3 \mathrm{~h}$ with $45 \%$ methanol, $45 \% \mathrm{H}_{2} \mathrm{O}, 10 \%$ glacial acetic acid. Primary antibodies used were for Tsg 101 (1:500, Abcam, Cambridge, UK; RRID: AB_1271357), CD9 (1:100, BioLegend, London, UK; AB_314907), mitofilin (1:500) and Grp78 (1:500, Proteintech, Manchester, UK; AB_2119855), TDP-43 (1:500, Proteintech, Manchester, UK; AB_615042), Src (1:200, Cell Signaling Technology, Leiden, The Netherlands, AB_2106059).

\section{RNA-Seq}

Vesicular samples (fractions 7-9) were pooled and incubated with $0.4 \mu \mathrm{g} / \mu \mathrm{l}$ RNase A (Sigma-Aldrich) for $10 \mathrm{~min}$ followed by extraction of RNA with the Arcturus PicoPure RNA Isolation Kit (Thermo Fisher) and oligo (dT) primed cDNA synthesis using the SMART-Seq v4 Ultra Low Input RNA Kit for Sequencing (Takara Bio, Saint-Germain-enLaye, France). Adapter indices were used to multiplex libraries, which were pooled prior to cluster generation using a cBot instrument. The loaded flow-cell was then paired-end sequenced $(76+76$ cycles, plus indices) on an Illumina HiSeq4000 instrument. Finally, the output data was demultiplexed (allowing one mismatch) and BCL-to-Fastq conversion performed using Illumina's bcl2fastq software, version 2.17.1.14.

\section{Bioinformatics}

The paired-end RNA-seq reads were quality assessed using FastQC (v 0.11.3), FastQ Screen (v 0.9.2). Mean Phred scores across the short reads were greater than 38 . Reads were processed with Trimmomatic (v 0.36) (to remove technical sequences and poor quality bases) In neurons, $13 \%$ of bases were removed and in EVs this was $28 \%$. The RNAseq reads were mapped against the reference human genome (hg38) using STAR (version 2.5.3a). Counts per gene were 
calculated with STAR using annotation from GENCODE (v27). Normalisation and differential gene expression analysis was performed using DESeq2 (using Benjamini-Hochberg correction for false discovery). The mean library size for neurons was $5 \times 10^{7}$ with a mapping rate of $1.5 \times 10^{8}$ reads per hour. The corresponding figures for EV libraries are $2.5 \times 10^{7}$ and $5.2 \times 10^{7}$. As the reads are different between cells and EVs (i.e. greater in cells, the data were sub-sampled to equalise the number of reads. This did not lead to a significant relative diminution of gene abundance in the cells as determined by linear regression analysis on the original and sub-sampled datasets nor substantial changes between EV and cell samples as determined by principal component analysis. RNA-seq data have been deposited in the ArrayExpress database at EMBL-EBI (www.ebi.ac.uk/ arrayexpress) under accession number E-MTAB-8254.

Ingenuity Pathways Analysis (IPA, Qiagen, Hilden, Germany) was used to explore gene networks for hypothesis generation. For mRNA, analysis thresholds were set at $\log _{2}$ fold change $>1$ and $q \leq 0.05$. Core analysis was based on $\log _{2}$ fold changes and the IPA knowledge database filtered to only include experimental findings in mammalian systems, limited to brain, primary neurons or neuroblastoma cells. Networks were limited to 70 focus molecules.

Genes were ordered by descending $\mathrm{p}$ value and the top 150 queried against UTRdb [28] to establish 5' and 3' UTR length. GC content was established using GCevobase [29] (querying 18,104 genes), predicted G quadruplexes using the EuQuad module of Quadbase 2 (target $\mathrm{G}_{\mathrm{x}} \mathrm{L}_{1-\mathrm{y}} \mathrm{G}_{\mathrm{x}} \mathrm{L}_{1-\mathrm{y}} \mathrm{G}_{\mathrm{x}} \mathrm{L}_{1-\mathrm{y}} \mathrm{G}_{\mathrm{x}}$ where $\mathrm{x}=3, \mathrm{y}=7$ and $\mathrm{L}$ is any base; querying top 1530 genes as organised by descending $p$ value) [30] and poly (A) tail length (querying 3863 genes available from TAIL-seq) from TAIL-seq analysis [31]. Data were analysed by D'Agostino-Pearson test for Gaussian distribution and Spearman's rank correlation.

\section{Quantitative PCR (qPCR)}

qPCR reactions were prepared as follows (total $20 \mu \mathrm{l}$ ): $1 \mu \mathrm{l}$ cDNA, $500 \mathrm{nM}$ each of forward and reverse primers with iQ Supermix (Bio-Rad). Thermal cycler (QuantStudio 3, Applied Biosystems, Thermo Fisher Scientific) parameters were set as follows: $3 \mathrm{~min} @ 95^{\circ} \mathrm{C}$ and forty cycles of $15 \mathrm{~s}$ @ $95{ }^{\circ} \mathrm{C}$ then $45 \mathrm{~s} @ 56{ }^{\circ} \mathrm{C}$. Primers were for PSEN2 (F: TCCTCAACTCCGTGCTGAAC; R: GCAGCGGTACTT GTAGAGCA), ATXN2 (F: TAATGACGACACAGCCAC CC, R: TAGGGGAAATGCGCTGTTGT); CHRNA7 (F: CGGCAAGAGGAGTGAAAGGT, R: AGGCCATAGTAG AGCGTCCT); HNRNPA1 (F: GATCCAAACACCAAG CGCTC, R: CCTTGTGTGGCCTTGCATTC); PICALM (F: GCCAAACTCCCACCTAGCAA, R: TGGTTCCAT TTCCGATGCCA)).

\section{Mass Spectrometry}

Vesicular fractions (fractions 7-9) were pooled and subjected to SDS-PAGE alongside equivalent total amounts of cellular protein. Electrophoresis was terminated once all sample had entered the resolving gel, such that proteins were present as a single band. Gel tops were stained with Coomassie Blue, destained and the protein band excised from the gel and dehydrated using acetonitrile followed by vacuum centrifugation. Dried gel pieces were reduced with $10 \mathrm{mM}$ dithiothreitol and alkylated with $55 \mathrm{mM}$ iodoacetamide. Gel pieces were then washed alternately with $25 \mathrm{mM}$ ammonium bicarbonate followed by acetonitrile. This was repeated, and the gel pieces dried by vacuum centrifugation. Samples were digested with trypsin overnight at $37^{\circ} \mathrm{C}$. Digested samples were analysed by LC-MS/MS using an UltiMate ${ }^{\circledR} 3000$ Rapid Separation LC (RSLC, Dionex Corporation, Sunnyvale, CA, USA) coupled to an Orbitrap Elite (Thermo Fisher Scientific, Waltham, MA, USA) mass spectrometer. Peptide mixtures were separated using a gradient from $92 \% \mathrm{~A}(0.1 \% \mathrm{FA}$ in water $)$ and $8 \% \mathrm{~B}(0.1 \% \mathrm{FA}$

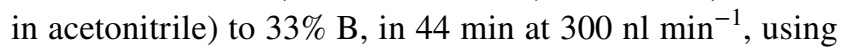
a $75 \mathrm{~mm} \times 250 \mu \mathrm{m}$ i.d. $1.7 \mathrm{mM}$ CSH C18, analytical column (Waters, Elstree, Herts, UK). Peptides were selected for fragmentation automatically by data dependant analysis. Data produced were searched using Mascot (Matrix Science UK), against the SwissProt_2018_01 database and validated using Scaffold (Proteome Software, Portland, OR, USA). The mass spectrometry proteomics data have been deposited to the ProteomeXchange Consortium via the PRIDE partner repository with the dataset identifier PXD015255.

\section{Data Analysis}

All experiments are $n=3$ unless otherwise indicated. Graphs were prepared using GraphPad Prism 7 (GraphPad Software, Inc., La Jolla, CA, USA).

\section{Results}

\section{Small EVs Can be Isolated from iPSC-Derived Neurons}

Neurons were derived from iPSCs as previously described [25-27]. The differentiated neurons express markers exclusive to mature neurons (including $\beta I I I$ tubulin and Tbr1), are electrophysiologically active from day 49 and form synapses as evidenced by visualisation of protein complex of synaptophysin/ PSD-95 and Munc13/ Homer [27]. Human iPSCs were confirmed as pluripotent (Fig. 1a) and subsequently differentiated into neurons (Fig. 1b and Fig. S1). The conditioned medium from human iPSC-derived neurons was subjected to 
(a)

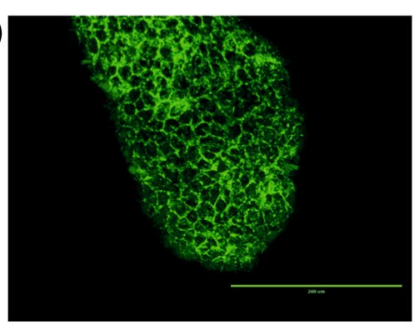

SSEA4

(b)

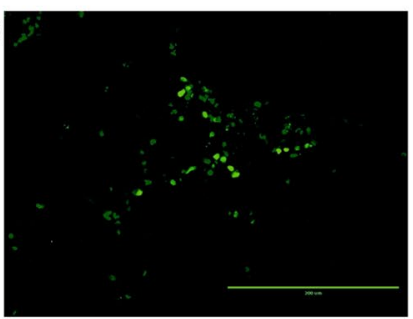

Satb2

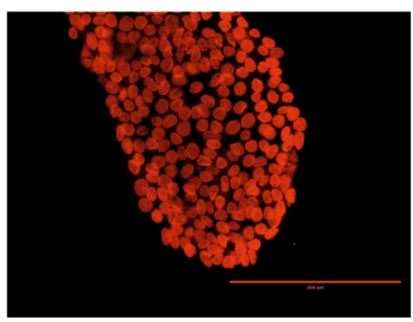

Oct4

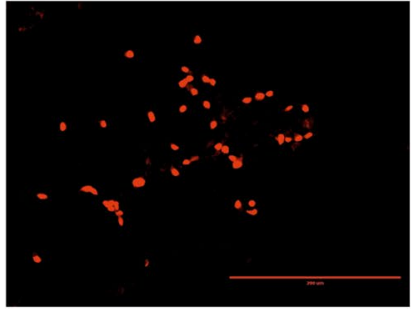

Tbr1

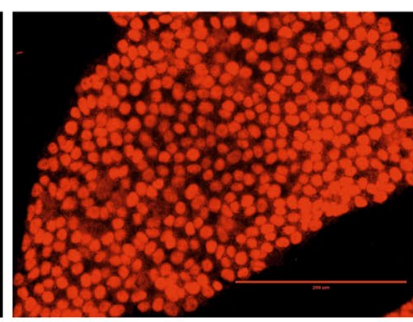

Sox2

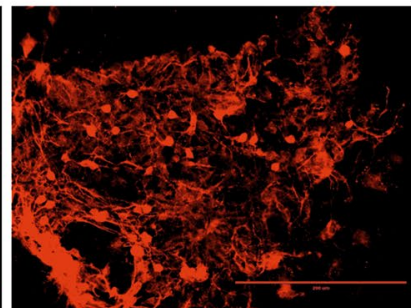

MAP2

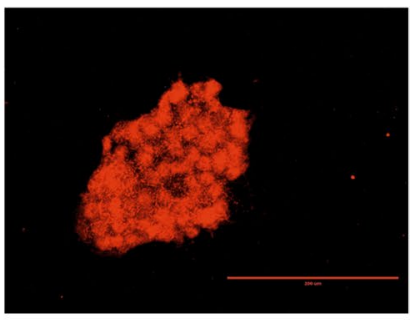

Nanog

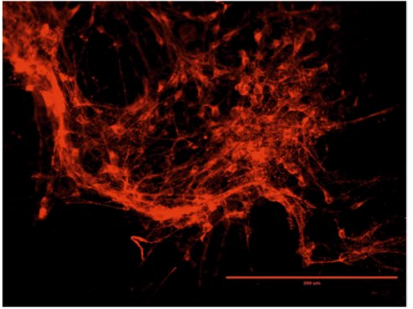

BIII tubulin
Fig. 1 Differentiation of iPSCs to neurons. a iPSCs were cultured as described in Experimental Procedures, fixed with 4\% paraformaldehyde and immunocytochemistry performed using antibodies against the pluripotency markers SSEA4, Oct4, Sox2 and Nanog. b iPSCs

size exclusion chromatography to isolate EVs. EM analysis confirmed a mean vesicle size of $50 \mathrm{~nm}$ (Fig. 2a, b). Dynamic light scattering was performed on individual fractions eluted from the size exclusion chromatography column (Fig. 2c). Fraction 6 represents the final $0.5 \mathrm{ml}$ of the void volume and vesicles were absent from this fraction. Vesicles were detected in fractions 7-10 and the mean particulate diameter decreased between successive fractions, from $77 \mathrm{~nm}$ in fraction 7 to $67 \mathrm{~nm}$ in fraction $8,46 \mathrm{~nm}$ in fraction 9 and $23 \mathrm{~nm}$ in fraction 10 (Fig. 2c). Immunoblotting for the small EV markers Tsg101 and CD9 was performed (Fig. 2d, e). There was minimal immunoreactivity towards Tsg101 in fractions 6 and 7, with overall immunoreactivity highest in fractions $8-10$. There was also Tsg101 immunoreactivity in fractions 11-13, which are non-vesicular fractions. Bulk protein was present in these fractions (Fig. S2), which may derive from EVs lysed during isolation or represent proteins present as large extracellular complexes. The pattern of Tsg101 immunoreactivity across the SEC fractions were mirrored by CD9. The ER chaperone Grp78 was not detected in the EV fractions (Fig. 2d). Similarly, there was only very weak immunoreactivity for the large vesicle marker mitofilin (Fig. 2d).

\section{A Subgroup of Cellular mRNA Transcripts are Selectively Incorporated into Neuronal EVs}

In order to characterize the mRNA cargo of neuronal sEVs, RNA-seq was performed on the iPSC-derived were differentiated to neurons as described in Experimental Procedures, fixed and immunocytochemistry performed using antibodies against the neuronal markers Satb2, Tbr1, MAP2 and $\beta$ III tubulin. Scale bar $=200 \mu \mathrm{m}$

neurons and their EVs. Comparison of mRNA transcript abundance between neurons and EVs showed a strong positive correlation, although using mean abundance to divide transcripts into quadrants showed a population of transcripts whose abundance was enriched in EVs (upper left quadrant; Fig. 3a). Taking the 500 most abundant EV mRNA transcripts and plotting them against their rank abundance in neurons confirmed a strong positive correlation, suggesting non-specific uptake of the most abundant neuronal mRNA transcripts into EVs (Fig. 3b). The 30 most enriched EV transcripts were plotted against their abundance in neurons (Table 1). The resultant heat map showed the highly enriched EV mRNA transcripts to be predominantly in the lowest tertile in terms of neuronal abundance (Fig. 3c). A volcano plot showed a typical distribution, identifying those subsets of mRNAs that are significantly increased and those that are decreased in EVs (Fig. 3d). Overall, gene body analysis of the top 200 most enriched transcripts showed no difference in read coverage between neurons and EVs. Analysis of 5' UTR length, 3' UTR length, \%GC content, predicted G quadruplex abundance and poly(A) tail length showed that only \% GC content is correlated with EV enrichment. Those genes with the highest fold change between EV and neuron had significantly higher GC content (Fig. S3). These data indicate that the most highly enriched mRNAs incorporated into neuronal EVs are characterized by relatively increased GC content. 
Fig. 2 Isolation of EVs from iPSC-derived neurons. iPSCderived neurons were cultured as described and exosomes isolated from the conditioned medium by size exclusion chromatography (SEC). a, b The vesicular fractions (7-9) were ultracentrifuged $(100,000 \mathrm{~g}$, $90 \mathrm{~min}$ ) and the pellet fixed and subjected to electron microscopy. Red arrows indicate sEVs c EV diameter was calculated from EM images using ImageJ. d Unconcentrated SEC fractions (6-13) were assessed for particle diameter using dynamic light scattering. $\mathrm{ND}=$ not detected. e SEC fractions were concentrated tenfold and subjected to SDS-PAGE alongside cell lysate (CL), followed by immunoblotting for Tsg 101, Grp78,mitofilin and CD9. Vertical line in CD9 panel indicates distal lanes from the same gel. $\mathbf{f}$ Tsg101 and CD9 immunoreactivity was quantified and plotted against SEC fraction number to demonstrate enrichment (a) EM

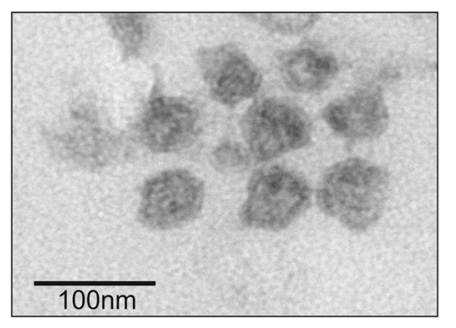

(c) DLS

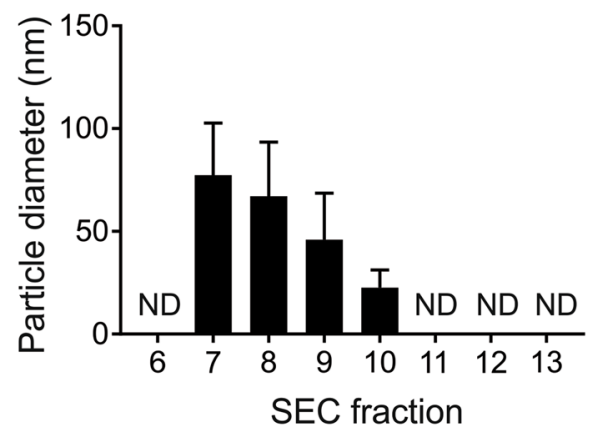

(e)

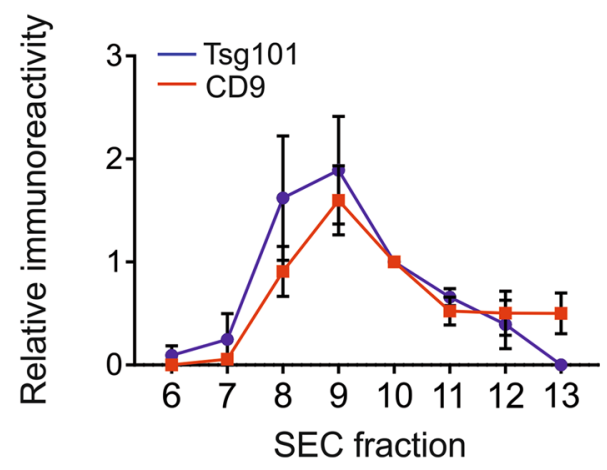

(b)

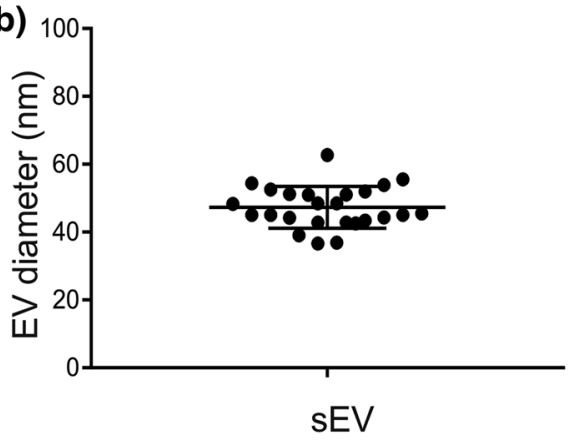

(d)

SEC fraction

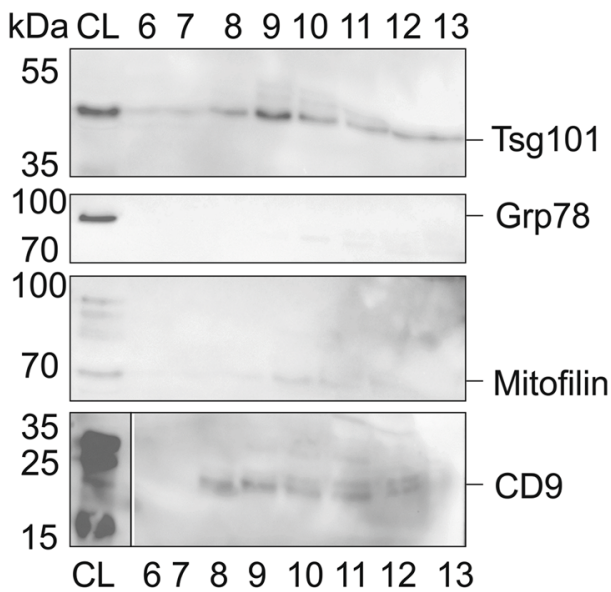

To elucidate potential functional roles of the most highly enriched neuronal EV mRNAs, Ingenuity Pathway Analysis (IPA) was used. Initially, physiological functions were probed, which revealed a strong focus on development and morphology, with 'Cellular Development' and 'Neurological Disease' featuring prominently (Fig. 4a and Table 2). Further analysis focusing on canonical signalling pathways showed EV-enriched mRNA transcripts to be linked to 'Agrin Interactions at the Neuromuscular Junction', 'Clathrin-mediated endocytosis' and various signalling pathways ('ATM Signaling', 'mTOR Signaling', 'actin cytoskeleton Signaling', 'BAG2 Signaling' and 'Integrinlinked kinase (ILK) Signaling' (Fig. 4b and Table 3). Although IPA outputs are based on experimentally validated data in primary neurons, brain or neuroblastoma cells, this does not imply that these processes are exclusive to neuronal EVs.

A transcriptional network was subsequently identified (Cell Morphology, Cellular Assembly and Organization, Nervous System Development and Function) which comprised $35 \mathrm{eV}$-enriched mRNA transcripts and was highly significant $\left(\mathrm{p}=10^{-34}\right)$ (Fig. 5 and Table 4). The network formed a hub and spoke structure, where hub transcripts are predominantly transcriptional regulators: MDM2, FMRI and JUN. (Fig. 5). Furthermore, several of the canonical signalling pathways identified in Fig. 4b mapped onto this network: mTOR signalling, ATM signalling, BAG2 signalling, agrin interactions at the neuromuscular junction, actin cytoskeleton signalling, clathrin-mediated endocytosis and ILK (Fig. 5). 
Fig. 3 A subgroup of cellular mRNA transcripts are selectively incorporated into neuronal EVs. mRNA was extracted from iPSC-derived neurons $(\mathrm{n}=3)$ and EVs $(\mathrm{n}=2)$, followed by RNA Seq and bioinformatics as described. a Abundance of specific mRNA transcripts was compared in neurons and EVs (red line at mean, green line shows linear regression), followed by $\mathbf{b}$ rank comparison for the 500 most abundant mRNA transcripts in EVs (green line shows linear regression, dotted line shows 95\% confidence) and c comparison of the top 30 most enriched mRNA transcripts in EVs against their rank abundance in neurons. d Volcano plot of all mRNA transcripts. Blue dotted line indicates the $\log _{2}$ fold change analysis threshold $\left(\log _{2}\right.$ fold change $>1$ ) and orange dotted line denotes the $-\log _{10}$ (p value) threshold (- $\log _{10}$ (p) $>2$ ). n number refers to an independent induction of iPSCs to neurons, from each a $\mathrm{sEV}$ preparation was isolated (Color figure online) (a)

(b)

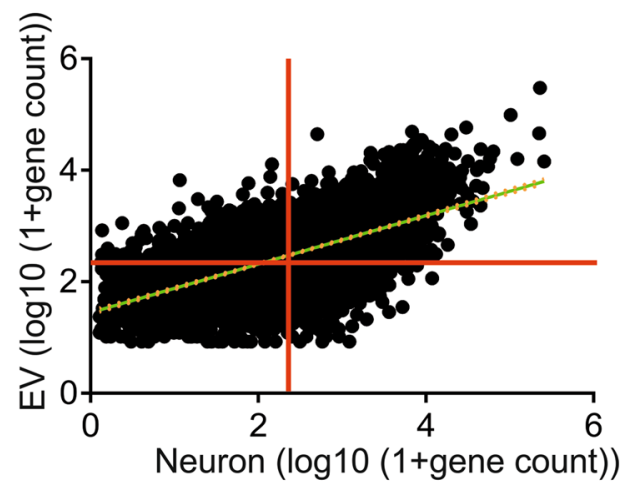

(c)

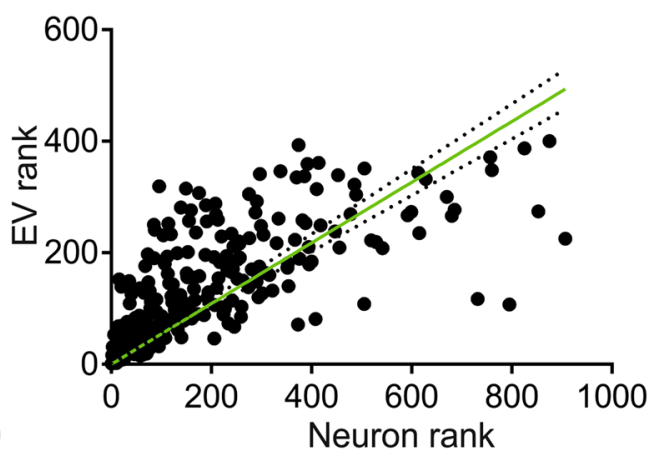

(d)

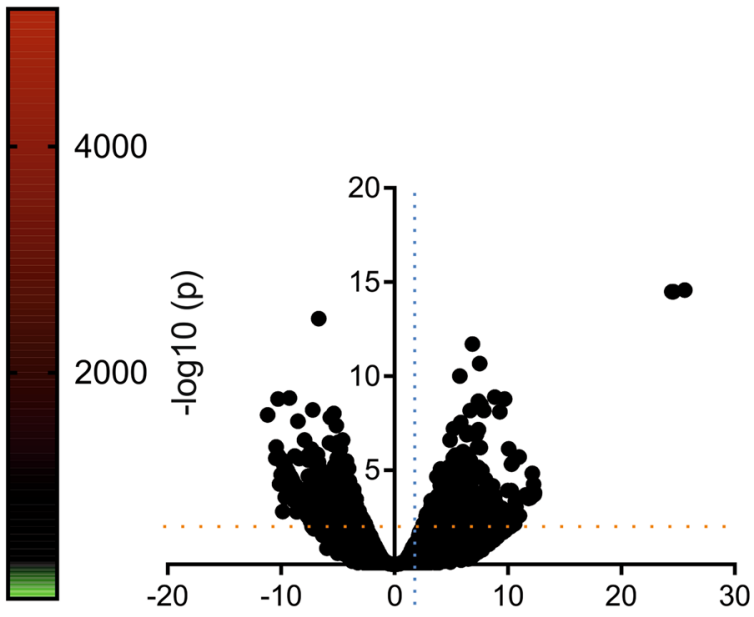

$\log 2$ fold change (EV:neuron)
Neuron EV enrichment rank rank
In some cases, these canonical pathways were linked by common signalling molecules, specifically $V E G F B$ (mTOR signalling, ILK signalling and clathrin-mediated endocytosis signalling), MDM2 (ATM signalling, BAG2 signalling and clathrin-mediated endocytosis signalling), JUN (Agrin interactions, ILK signalling and ATM signalling) and PFNI (actin cytoskeleton and clathrin-mediated endocytosis signalling). Together, these analyses show that neuronal EVs contain a putative transcriptional network with key hub genes that connect multiple downstream signalling pathways. In addition, the IPA networks were assessed for inter-connectivity, showing that the networks are predominantly distinct, with few overlapping genes (Fig. S4). Enriched transcripts with links to neuronal function and also dysfunction in neurodegeneration were validated by qPCR: ATXN2, CHRNA7, HNRNPA1, PICALM and PSEN2. The presence of these mRNAs was confirmed in EVs (Fig. S5.)

\section{Proteomic Analysis of Neuronal EVs Identifies Enrichment of Cellular Maintenance and Endocytic Signalling Pathways}

To complement the transcriptomic analysis of neuronal EVs, proteomic mass spectrometry analysis was also performed, followed by IPA on the EV-enriched proteins. Proteomic analysis was able to identify CD9 in the EV sample, but not in the cell fraction. This does not imply absence of expression in neurons, but can be inferred as enrichment in EVs. Several of the IPA categories from this analysis correlated well with the transcriptomic analysis. Common categories included 'Nervous System Development and Function', 'Cell Death and Survival', 'Cell-To-Cell Signalling and Interaction', alongside other morphological and developmental functions (Fig. 6a and Table 5). Investigation of the canonical signalling pathways linked to EV-enriched proteins revealed enrichment of 'Agrin Interactions at Neuromuscular 
Table 1 Top 30 most enriched genes in EVs and rank expression in neurons

\begin{tabular}{|c|c|c|c|}
\hline Gene & Neuron rank & $\begin{array}{l}\text { EV enrichment } \\
\text { rank }\end{array}$ & $\begin{array}{l}\text { mRNA } \\
\text { length } \\
\text { (bases) }\end{array}$ \\
\hline CCDC9 & 148 & 1 & 2027 \\
\hline KCNE3 & 3011 & 2 & 3143 \\
\hline RASSF3 & 3983 & 3 & 3507 \\
\hline ABCA4 & 4897 & 4 & 7328 \\
\hline SLC14A2 & 4804 & 5 & 4073 \\
\hline TNFSF4 & 3222 & 6 & 3492 \\
\hline SVEP1 & 2104 & 7 & 12,205 \\
\hline CTXN1 & 4524 & 8 & 1237 \\
\hline SNAI2 & 2352 & 9 & 2180 \\
\hline CYLC2 & 4922 & 10 & 2149 \\
\hline SEC14L3 & 5011 & 11 & 2086 \\
\hline SLC6A2 & 3138 & 12 & 2471 \\
\hline MX2 & 5120 & 13 & 3408 \\
\hline ANP32B & 224 & 14 & 1483 \\
\hline KNG1 & 5209 & 15 & 4198 \\
\hline TNN & 5040 & 16 & 5042 \\
\hline PRPH & 1912 & 17 & 1800 \\
\hline ITIH5 & 4734 & 18 & 6716 \\
\hline PLEKHA4 & 434 & 19 & 3073 \\
\hline CLDN11 & 5196 & 20 & 2169 \\
\hline KIF1C & 474 & 21 & 7917 \\
\hline CHIT1 & 4595 & 22 & 2248 \\
\hline CPNE9 & 4179 & 23 & 2042 \\
\hline C6orf141 & 1475 & 24 & 1450 \\
\hline PAQR7 & 3633 & 25 & 3297 \\
\hline SCLY & 3142 & 26 & 2526 \\
\hline TRDN & 3354 & 27 & 1294 \\
\hline FBF1 & 2437 & 28 & 3626 \\
\hline GALNT6 & 4745 & 29 & 5307 \\
\hline CENPP & 5064 & 30 & 2570 \\
\hline
\end{tabular}

Using RNA seq data, ratios of mRNA abundance were calculated (EV: neuron) and those mRNA transcripts with the highest enrichment were tabulated and compared to their rank abundance in neurons

Junction' and 'Glycoprotein VI (GP6) Signalling Pathway' (Fig. 6b and Table 6). The latter is usually restricted to platelets, but IPA has likely identified this pathway due to substantial enrichment in collagen and laminin isoforms in neuronal EVs. Several signalling and cell entry pathways were also implicated in this analysis: 'Clathrin-mediated Endocytosis Signalling' and 'Caveolar-mediated Endocytosis Signalling', in addition to 'CDK5 Signalling', Ephrin Receptor Signalling', 'focal adhesion kinase (FAK) Signalling', 'Integrin Signalling' and 'Neuregulin Signalling' (Fig. 6b and Table 6). Together with the transcriptomic data, these proteomic data indicate that neuronal EVs contain proteins that can exert downstream effects via cell surface interactions and also through cell entry. Two proteins were selected for further immunoblot validation, based on their centrality in signalling pathways, either as a master regulator of cell signalling $(\mathrm{Src})$ or a regulator of thousands of RNA targets (TDP-43). Immunoblotting confirmed the presence of both proteins in EVs (Fig. S5).

\section{Discussion}

As non-coding RNA generally predominates in EVs, the minority RNA subtypes, including mRNA, have not been well studied $[32,33]$. There are relatively few reports of RNA-seq in sEVs [33-35] and this study is the first in iPSCderived neurons. Although there was a strong positive correlation between mRNA abundance in the neurons and their EVs, the most highly enriched EV mRNA transcripts on the whole were less abundant in the neurons, implying that a subgroup of mRNAs are selectively enriched in the EVs. Though mRNA requires translation for functionality, there are several reports of the ability of mRNAs to enter and be translated in recipient cells [7, 8, 32]. In agreement with previous reports, our RNA-seq analysis highlights the large number of mRNA transcripts encoding ribosomal subunits enriched in the neuronal EVs [34]. This may indicate that EVs contain mRNA encoding elements of the translational machinery to facilitate expression of EV mRNA. Other key IPA categories most strongly linked to EV RNA were those involved in cell development and survival. These are well reported functions of EVs [36-40]. Canonical pathway analysis also implicated integrin-linked kinase (ILK) signalling. Integrin signalling is strongly linked to EV function [2, 41] and is likely to play a role in EV-mediated signalling.

Proteomic analysis of the neuronal EVs concurred with the transcriptomic analysis findings; development, cell to cell signalling, morphology and cell growth/ proliferation were also prominent IPA categories. Additional foci of the proteomics analysis were cell interaction and entry pathways, specifically clathrin- and caveolar-mediated endocytosis, both of which have been linked to EV cell entry [42-45]. Integrins were prominent in the proteomic analysis and integrin signalling was a key IPA term. Integrins are strongly linked to EV-cell interactions and cell entry $[41,46]$. Lastly, agrin interactions at the neuromuscular junction featured in the IPA for proteins and mRNA, which suggests that neuronal EVs may impact on the organisation of synapses [47].

Overall, the bioinformatics analysis of the neuronal EV transcriptomic and proteomic data segregates into four cellular processes: (i) cell entry pathways and cell surface signalling; (ii) intracellular downstream signalling; (iii) specific functional outcomes (e.g. activation of key signalling pathways); and (iv) general phenotypic outcomes (e.g. 
Fig. 4 Developmental signalling networks are enriched in the mRNA from neuronal EVs. Following RNA-seq analysis, mRNA transcripts enriched in neuronal EVs $(\mathrm{p}<0.01)$ were processed by Ingenuity Pathway Analysis (IPA) and reported by a physiological function and $\mathbf{b}$ canonical signalling pathways. The horizontal bar indicates the range, where the central vertical line indicates the mean

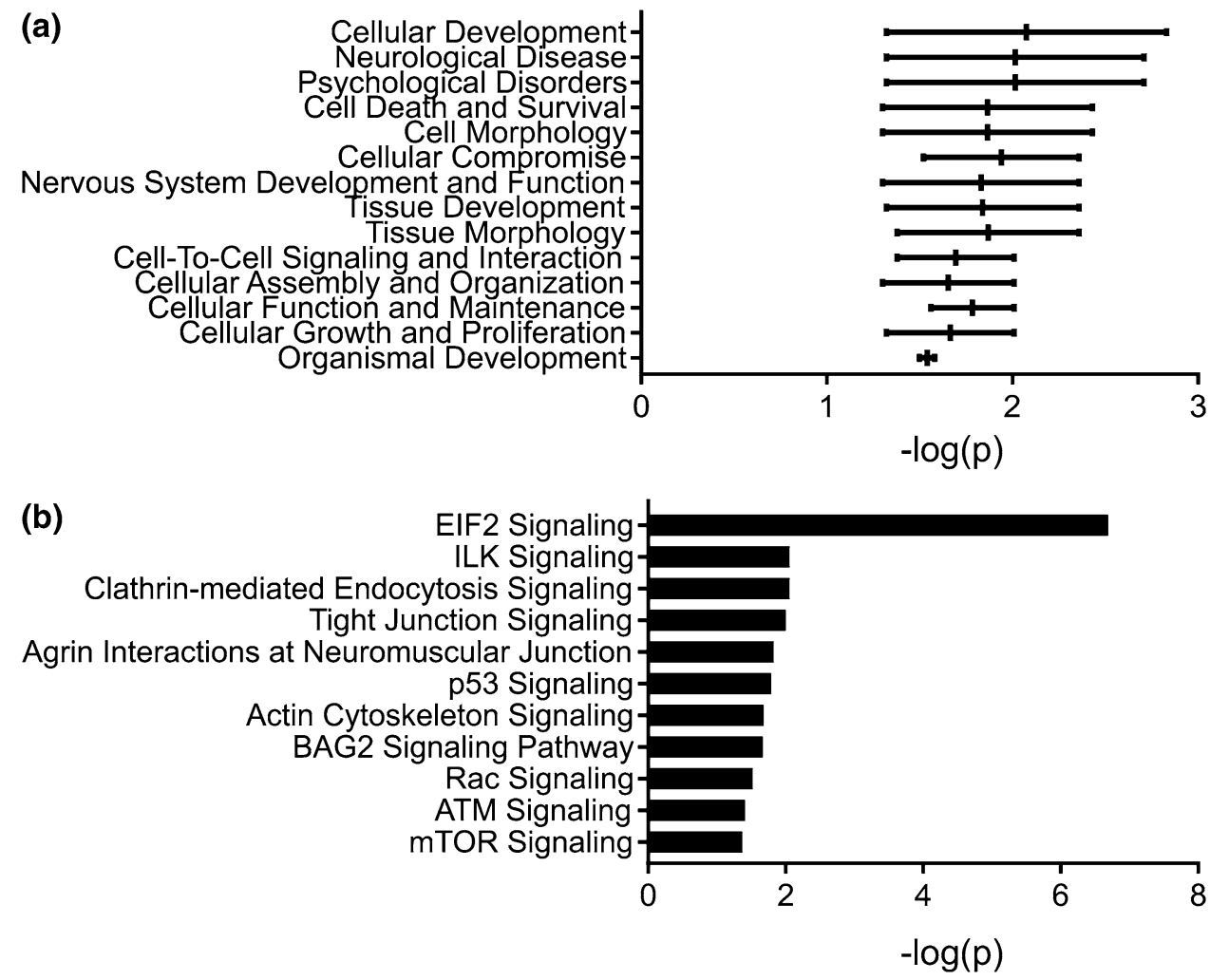

(a)

(b)

$-\log (p)$

'Nervous System Development and Function' and 'Cellular Organisation and Assembly'). Taking these cellular processes together one could speculate that neuronal EVs interact with neighbouring cells via integrins, collagens, neuregulin and the ephrin receptors and are internalised via clathrin- and caveolin-dependent mechanisms followed by downstream signalling Roles for ephrin receptor, p53, ILK and MAPK signalling have been recently identified for EVs [48-52]. These downstream signalling pathways can lead to synaptic modification through agrin interactions, which may lead to pronounced effects on cellular development and maintenance, aligning with previous reports [53,54]. These latter effects may be driven, at least in part, by putative transcriptional networks such as the one identified here (Fig. 6).
Although individual iPSC-derived neurons are highly representative of mature human neurons [25-27], there are limitations, such as the uncertain status of epigenetic modifications after reprogramming and maturation [55]. Similarly, cells in this study were cultured in an in vitro 2-D monoculture, which lacks the support of the extracellular matrix and other cell types of the neurovascular unit [56] and the effect of these factors on RNA loading into EVs remains unclear.

In conclusion, exploratory transcriptomic and proteomic analysis of EVs secreted by human iPSC-derived neurons has identified key facets of neuronal $\mathrm{EV}$ function, which may link to signalling pathways related to EV entry into cells and key downstream functional effects. This supports previous work showing the ability of EV RNA to enter target cells and modify their phenotype [57-60]. 
Table 2 Ingenuity pathway analysis (IPA) gene lists (highly enriched EV mRNA transcripts were selected (q value $<0.05)$ and assigned to physiological)

\begin{tabular}{|c|c|c|}
\hline Function & $-\log _{10} \mathrm{p}$ value & Genes \\
\hline Cellular development & $1.32-2.83$ & $\begin{array}{l}\text { ACHE,ACTN1,BAG1,CEACAM1,CLN3,DGCR8,DISC1,EMC10,ERBB3,FMR1, } \\
\text { GLIPR1,HIPK2,HNF4A,KNG1,LHX4,LIMK1,MEF2A,NOP53,NOS1,PDGFA,P } \\
\text { FN1,PIAS2,PPP1R9B,PTPN11,RUNX1,SCLY,SLC9A3R1,THRB,UNC13A }\end{array}$ \\
\hline Neurological disease & $1.32-2.71$ & $\begin{array}{l}\text { ACTA2,AEBP1,ASPA,ATF3,BAG1,BBC3,BCAS1,CCDC80,CCN2,CD44,CHRN } \\
\text { A7,CIC,CLN3,CLN5,ERBB3,JUN,KNG1,LAMB1,LAMP1,LHX4,MDM2,MEC } \\
\text { OM,MYO15A,MYO1B,NOS1,PFN1,PIAS2,PRPH,PTPN11,RIPK2,RPL13,RSF } \\
\text { 1,S100A10,SCLY,TPT1,TRAK2,TRIB3,TRIM56,UNG,VEGFB,VIM,WWTR1 }\end{array}$ \\
\hline Psychological disorders & $1.32-2.71$ & $\begin{array}{l}\text { AEBP1,ATF3,BBC3,BCAS1,CCN2,CD44,CLN3,JUN,KNG1,LAMB1,LAMP1,M } \\
\text { YO1B,NOS1,PFN1,PIAS2,PRPH,RIPK2,RPL13,S100A10,TPT1,TRAK2,TRIB } \\
\text { 3,VIM,WWTR1 }\end{array}$ \\
\hline Cell death and survival & $1.30-2.43$ & $\begin{array}{l}\text { ATF3,CD44,ERBB3,HIPK2,JUN,NOS1,PRPH,PTPN11,RIPK2,RUNX1,SCLY,SH } \\
\text { 3KBP1,SNAI2,UNG }\end{array}$ \\
\hline Cell morphology & $1.30-2.43$ & $\begin{array}{l}\text { ACHE,ATF3,CABP4,CHRNA7,CLN3,CLN5,DISC1,DOCK10,ERBB3,FMR1,GA } \\
\text { TA3,GDF11,HHAT,HIPK2,HSPA4,LHX4,LIMK1,LMNA,MDM2,MSRB3,MY } \\
\text { O15A,NOS1,NYAP1,PARD6A,PFN1,RIPK2,RUNX1,S1PR2,SHANK2,SLITRK } \\
\text { 5,SNAI2,TGFB3,THRB,TLR7,UNC13A,UTRN,VIM }\end{array}$ \\
\hline Cellular compromise & $1.52-2.36$ & ASPA,JUN,PIK3CG,SCLY,TRIB3,UNG \\
\hline Nervous system development and function & $1.30-2.36$ & $\begin{array}{l}\text { ACHE,ATF3,BAG1,CABP4,CHRNA7,CLN3,CLN5,DGCR8,DISC1,DOCK10,ER } \\
\text { BB3,FMR1,GATA3,GDF11,HHAT,HIPK2,HSPA4,JUN,KNG1,LHX4,LIMK1,L } \\
\text { MNA,MDM2,MECOM,MEF2A,MYO1B,NOS1,NYAP1,PARD6A,PFN1,PIAS2, } \\
\text { PTPN11,RIPK2,RUNX1,S1PR2,SCLY,SHANK2,SLC24A4,SLITRK5,TGFB3,T } \\
\text { HRB,TLR7,UNC13A,UTRN,VEGFB,VIM }\end{array}$ \\
\hline Tissue development & $1.32-2.36$ & $\begin{array}{l}\text { CEACAM1,DISC1,ERBB3,FMR1,HIPK2,MEF2A,NOS1,PFN1,PIAS2,PTPN11,R } \\
\text { UNX1,S1PR2,SCLY,UNC13A }\end{array}$ \\
\hline Tissue morphology & $1.38-2.36$ & $\begin{array}{l}\text { ACHE,CABP4,CHRNA7,CLN3,CLN5,DISC1,DOCK10,ERBB3,FCMR,FMR1,G } \\
\text { ATA3,GDF11,HHAT,HIPK2,HSPA4,JUN,KNG1,LHX4,LIMK1,LMNA,MDM2 } \\
\text {,NOS1,NYAP1,PARD6A,PFN1,RUNX1,SCLY,SHANK2,SLITRK5,TGFB3,TH } \\
\text { RB,TLR7,UNC13A,UNG,UTRN,VIM }\end{array}$ \\
\hline Cell-to-cell signaling and interaction & $1.38-2.01$ & $\begin{array}{l}\text { ACHE,CD44,CHRNA7,DISC1,FMR1,HIPK2,KNG1,PFN1,SELP,SHANK2,SLC } \\
\text { 24A4,SLITRK5 }\end{array}$ \\
\hline Cellular assembly and organization & $1.30-2.01$ & $\begin{array}{l}\text { ATF3,CBX1,CEACAM1,CLN3,DISC1,FMR1,HIPK2,NOS1,NYAP1,PFN1,RIPK } \\
\text { 2,S1PR2,SLITRK5,UTRN }\end{array}$ \\
\hline Cellular function and maintenance & $1.56-2.01$ & ACHE,CEACAM1,CLN3,DISC1,FMR1,HIPK2,NOS1,PFN1,S1PR2 \\
\hline Cellular growth and proliferation & $1.32-2.01$ & $\begin{array}{l}\text { ACHE,ACTN1,BAG3,CEACAM1,CHRNA7,CLN3,DISC1,EMC10,ERBB3,FMR } \\
\text { 1,GLIPR1,HIPK2,HNF4A,KNG1,LIMK1,NOP53,NOS1,PDGFA,PFN1,PPP1R9 } \\
\text { B,PTPN11,SLC5A8,SLC9A3R1,THRB }\end{array}$ \\
\hline Organismal development & $1.50-1.58$ & $\begin{array}{l}\text { ACHE,ASPA,ATXN1L,CABP4,CHRNA7,CLN3,CLN5,ERBB3,FMR1,FSCN1,G } \\
\text { ATA3,GDF11,HSPA4,ID1,JUN,KNG1,LAMP1,LHX4,LIMK1,MDM2,MECOM } \\
\text {,MSRB3,NOS1,NYAP1,PPP1R9B,SEMA3B,SHANK2,SLITRK5,THRB,TLR7, } \\
\text { VIM,ZFYVE26 }\end{array}$ \\
\hline
\end{tabular}


Table 3 IPA gene list for RNA-seq data (canonical pathways)

\begin{tabular}{lll}
\hline Pathway & $-\log _{10} \mathrm{p}$ value & Genes \\
\hline EIF2 signaling & 6.69 & $\begin{array}{c}\text { ACTA2,ACTG2,ATF3,PABPC1,PIK3CG,PPP1CC,PTBP1,RPL11,RPL12,RPL } \\
\text { 13,RPL23,RPL23A,RPL26,RPL37,RPL37A,RPL38,RPS12,RPS15A,RPS17, } \\
\text { RPS24,TRIB3 }\end{array}$ \\
& & $\begin{array}{c}\text { ACTA2,ACTG2,ARPC2,CLTA,EPN1,FGF23,MDM2,PDGFA,PIK3CG,SH3K } \\
\text { BP1,VEGFB }\end{array}$ \\
Clathrin-mediated endocytosis signaling & 2.06 & ACTA2,ACTG2,ACTN1,FLNB,JUN,KRT18,PIK3CG,PPP2R5A,SNAI2,VEG \\
ILK signaling & 2.06 & FB,VIM \\
& 2 & ACTA2,ACTG2,CLDN11,CNKSR3,JUN,PARD6A,PPP2R5A,RAB13,TGFB3 \\
Tight junction signaling & & ,YBX3 \\
& & ACTA2,ACTG2,ERBB3,JUN,LAMB1,UTRN \\
Agrin interactions at neuromuscular junction & 1.83 & BBC3,HIPK2,JUN,MDM2,PERP,PIK3CG,SNAI2 \\
p53 signaling & 1.79 & ACTA2,ACTG2,ACTN1,ARPC2,FGF23,KNG1,LIMK1,PDGFA,PFN1,PIK3C \\
Actin cytoskeleton signaling & 1.68 & G,TLN2 \\
& & CHRNA7,HSPA1A/HSPA1B,HSPA4,MDM2 \\
BAG2 signaling pathway & 1.67 & ARPC2,CD44,JUN,LIMK1,PARD6A,PIK3CG,PLD1 \\
Rac signaling & 1.52 & CBX1,H2AFX,JUN,MDM2,PPP1CC,PPP2R5A \\
ATM signaling & 1.41 & DGKZ,FKBP1A,PIK3CG,PLD1,PPP2R5A,RPS12,RPS15A,RPS17,RPS24,VE \\
mTOR signaling & 1.37 & GFB \\
& &
\end{tabular}




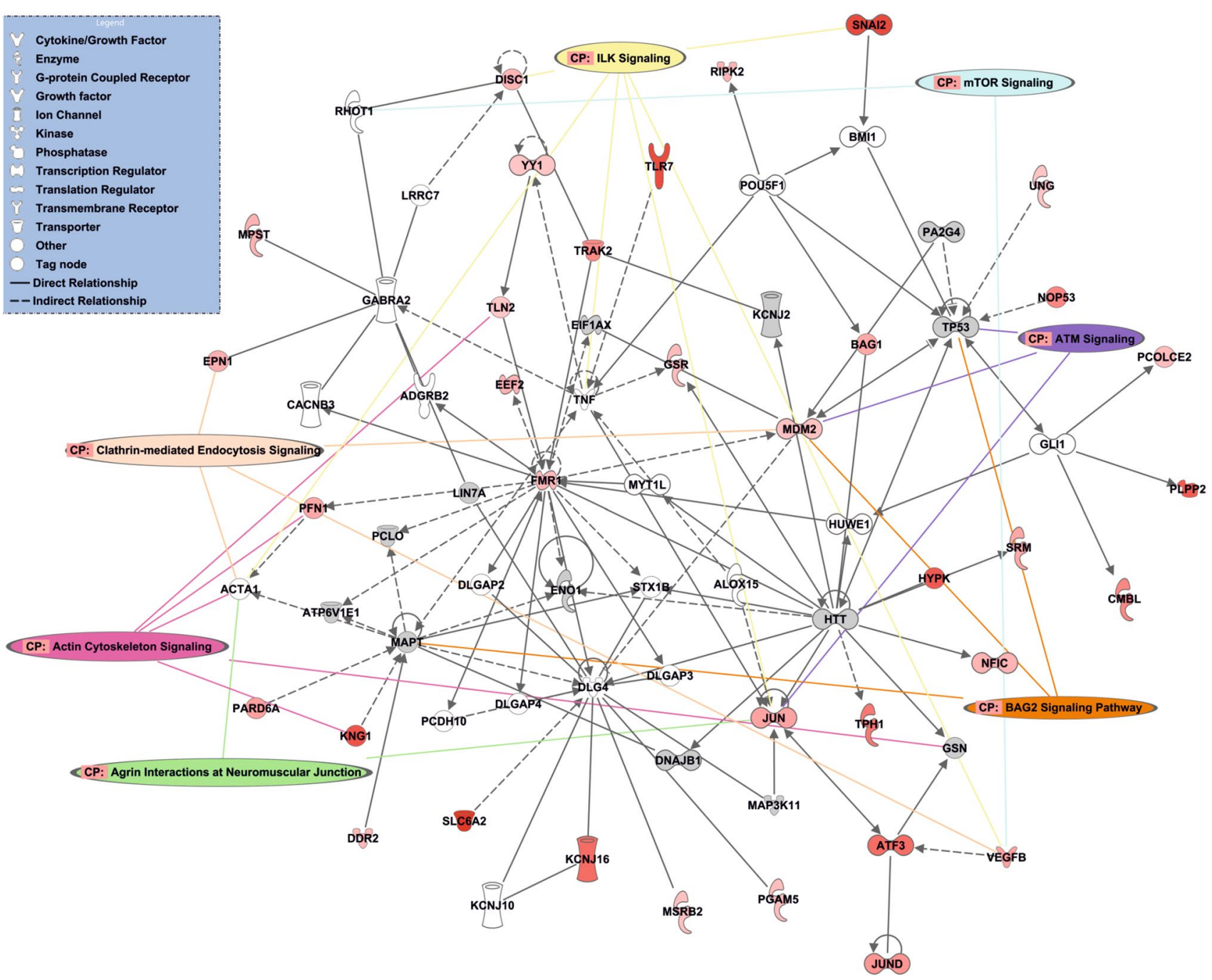

Fig. 5 Network analysis of mRNA in neuronal EVs. IPA core analysis was used to generate a network of significantly enriched mRNA transcripts $\left(\mathrm{p}=10^{-34}\right)$, using 35 focus molecules. Red intensity is proportional to $\log _{2}$ fold change, while grey indicates mRNA transcripts that did not reach the analytical cut off $(q \leq 0.01)$. White coloured molecules indicate network mediators that did not appear in the gene list supplied to IPA. Whole lines indicate direct relationships, while dotted lines indicate indirect relationships. Canonical pathways (CP) are mapped onto the network, connected to their cognate mRNA transcripts by coloured lines. Direct relationships are classed as experimental determined interactions; indirect interactions are predicted based on experimentally determined intermediate interactors (Color figure online) 
Table 4 IPA network list for RNA-seq data (putative signalling networks generated by IPA)

\begin{tabular}{ll}
\hline ID Function & Focus Score Genes \\
& mol- \\
& ecules
\end{tabular}

1 Cell morphology, cellular assembly and organization, nerv- 35 ous system development and function

34

ACTA1,ADGRB2,ALOX15,ATF3,ATP6V1E1,BAG1,BMI 1,CACNB3,CMBL,DDR2,DISC1,DLG4,DLGAP2,DLGA P3,DLGAP4,DNAJB1,EEF2,EIF1AX,ENO1,EPN1,FMR 1,GABRA2,GLI1,GSN,GSR,HTT,HUWE1,HYPK,JUN,J UND,KCNJ10,KCNJ16,KCNJ2,KNG1,LIN7A,LRRC7,M AP3K11,MAPT,MDM2,MPST,MSRB2,MYT1L,NFIC,N OP53,PA2G4,PARD6A,PCDH10,PCLO,PCOLCE2,PFN1 ,PGAM5,PLPP2,POU5F1,RHOT1,RIPK2,SLC6A2,SNAI 2,SRM,STX1B,TLN2,TLR7,TNF,TP53,TPH1,TRAK2,U NG,VEGFB,YY1

2 Cell death and survival, cellular assembly and organization, 31 cellular function and maintenance

Acetylcholine,ACHE,AGER,APC,APP,ARF6,ATF4,BAD,B BC3,BCL2L11,CAMK4,CCDC120,CCL3L3,CCN2,CFL 1,CHAT,CHRNA7,CITED2,CLN5,CLTA,COL4A3BP,CR TAP,CXCR4,DDAH1,DNM1L,FKBP1A,FLNA,FOXO1, GLUL,GNAS,GSR,HERC6,HGF,HSPA1A/HSPA1B,HSP A4,HSPB1,IDE,IGF1R,IRAK4,IRF3,IRF7,ITIH5,KCNE3 ,KIF5B,KIF5C,KLC1,KLC2,LIMK1,MAP2K4,MIF,NEN F,NF1,NFKB1,PAK1,PLD1,PRKCA,RFLNB,RNF144B, RPS6KB1,SH3PXD2A,SLC5A7,STIP1,SUMO2,TGFB3, THRB,TPT1,TRIB3,TSC1,UBQLN1,ZNRF1

3 Cell death and survival, protein synthesis

ADAM17,AEBP1,APBB1,AXL,BCL2L1, beta-estradiol,B IRC5,CASP8,CAV2,CDKN2A,CLDN11,COL1A1,CYP 19A1,DGKZ,EGF,EGFR,ERBB2,FN1,GNRH1,GRB2,H K2,HLA-A,ID1,IDE,IGF1R,IGFBP2,ITGB1,JUN,LAM A2,LAMB1,LEP,LEPR,MIR17HG,MSRB3,MYCN,MY OC,NME3,NOS1,NUMB,PABPC1,PDK1,PLP1,PPP1R9 B,PTK2,RARA,RGS5,RHBDF1,RPL11,RPL12,RPL13, RPL23,RPL23A,RPL26,RPL37,RPL37A,RPL38,RPS12, RPS17,RPS24,RPS6,SET,SIDT1,SLC2A1,SP1,STAT3,T GFA,TP73,TSHZ2,TSPAN3,VIM

4 Neurological disease, organismal injury and abnormalities, 28 skeletal and muscular disorders abnormalities
ACTA2,AGT,APBB1,ARC,ATXN1,ATXN1L,BAIA P2,CCDC80,CCL2,CD14,CTXN1,CYTH4,DLG2, DRD1,EGR1,EGR2,GAPDH,GDNF,GFAP,GRIA1, GRIA2,GRID2,GRIN2A,GRIN2B,GRM2,H2AFX,HMGB1,HMGB2,IL1B,IL6,L-dopa,LMNA,MAPK1,MA PK3,MXD1,MYD88,MYO1B,NET1,NEUROD6,NGF,N PM3,PDGFA,PGPEP1,PIK3CG,PITPNM3,PKM,PPARG C1A,PRKCG,PTBP1,PTGS2,PTPN4,RAB13,RIMS1,S10 0A10,S1PR2,SELP,SLC39A14,SLITRK5,SLMAP,SNCA ,SRF,SYN1,TH,TIRAP,TLR2,TLR4,TRIB3,UNC13A,W WTR1,YWHAE

ACSL5,ACTG2,ACTN1,ADAM10,BCL2,BIRC5,C2,Cc17, CCND1,CD44,CDH1,CDH5,CDK5,CDK5R2,CEACAM 1,CLEC2D,CRABP1,CTNNB1,CX3CL1,CXCL2,DGCR 8,DUSP1,E2F1,EGR2,ERBB3,FOXM1,GATA3,GDF11, GPRC5A,HES5,HNF4A,ID4,IL1R2,IL6ST,INS,ITGAM,J AG1,KIF1B,KMT2D,LAMA2,MSX1,MYO15A,NF2,NO TCH1,NOTCH3,NRG1,NT5DC1,PAK1,PER3,Pou3f1,PO U3F2,PPP1R13L,PRX,PSEN1,RGS10,SLC52A2,SMO,S MOC1,SOCS3,STAT3,SYK,TCF7,TGFB1,TGFBR3,TM EM40,TRPV1,TXNRD2,UTRN,WTIP,YAP1 
Table 4 (continued)

\begin{tabular}{|c|c|c|c|c|}
\hline ID & Function & $\begin{array}{l}\text { Focus } \\
\text { mol- } \\
\text { ecules }\end{array}$ & Score & Genes \\
\hline 6 & $\begin{array}{l}\text { Cellular development, cellular growth and proliferation, } \\
\text { nervous system development and function }\end{array}$ & 27 & 23 & $\begin{array}{l}\text { ADAM17,BAG3,BDNF,C16orf70,CAMK2D,CASK,Cdkn } \\
\text { 1c,CLN3,DGCR8,DNAJC21,DNM2,EFNA2,EFNA5,EIF } \\
\text { 2AK2,ELK1,EPO,FLNB,FSCN1,GNAI1,GRIA1,GRIN2 } \\
\text { B,HIPK2,HLA-A,HTR1A,HTR2C,Ins1,ITIH3,ITPR1,KI } \\
\text { F17,KIF1A,KIF3A,LAMB3,LIN7A,MAG,MAP3K11,M } \\
\text { APK8IP1,MEF2A,MEF2C,NF2,NGFR,NOS1,Nos1ap,N } \\
\text { RG1,NUDT16L1,NYAP1,PDE11A,PDGFRA,PDLIM1, } \\
\text { PPP1CC,PPP2R5A,PTEN,PTPN11,RAC1,RACK1,RASG } \\
\text { RF1,RYR2,S100A9,SEMA3B,SHANK2,SIM1,SIRPA,SI } \\
\text { RT1,SLC9A3R1,SLITRK5,SYNPO,TNIK,TPP1,TRAF3I } \\
\text { P1,WASF1,YWHAG }\end{array}$ \\
\hline 7 & $\begin{array}{l}\text { Cellular development, cellular movement, nervous system } \\
\text { development and function }\end{array}$ & 24 & 19 & $\begin{array}{l}\text { ANKRD13A,ARPC2,ASPA,BCAS1,BCL2,BEST1,CART } \\
\text { PT,CHD4,CLDN11,CNKSR3,CNP,corticosterone,CREB } \\
\text { 1,DBH,DGCR8,DOCK10,dopamine,FOXM1,GAB1,GIP } \\
\text { C1,GNAS,GSN,HES5,HIPK2,HTR2C,IL6R,IRS2,ISL1, } \\
\text { JAG1,KCND2,KCNK6,LAMP1,LIF,LMCD1,MCL1,MC } \\
\text { TP1,ME1,MET,MSI2,MYRF,NEUROD1,NR4A2,NRP1 } \\
\text {,OLIG1,OLIG2,PLCH2,PLEKHA4,POU4F1,PRKCE,PR } \\
\text { KCH,PRKCQ,PRL,PROM1,PRPH,QKI,RUNX1,S100A1 } \\
\text { 3,SEMA5A,SH3KBP1,SOX10,SOX2,SREBF1,STAT3,T } \\
\text { AC1,TAF10,TCF7L2,testosterone,TLR4,TRH,TRPV1 }\end{array}$ \\
\hline
\end{tabular}

Focus molecules was the number of EV-enriched mRNA transcripts included in the network. Score was the negative exponent of Fisher's Exact test result

Fig. 6 Cellular maintenance and endocytic signalling pathways are enriched in the proteome of neuronal EVs. Following proteomic analysis, proteins enriched in neuronal EVs (total counts ratio $>1$ in at least two biological replicates) were processed by IPA and reported by a physiological function and b canonical pathway. The horizontal bar indicates the range, where the central vertical line indicates the mean (a) Cellular Function and MaintenanceCellular Assembly and OrganizationNervous System Development and FunctionCellular Growth and ProliferationCellular Development Cell MorphologyNeurological DiseaseCell-To-Cell Signaling and InteractionSmall Molecule Biochemistry Molecular Transport

Amino Acid MetabolismCellular MovementCell Death and Survival-

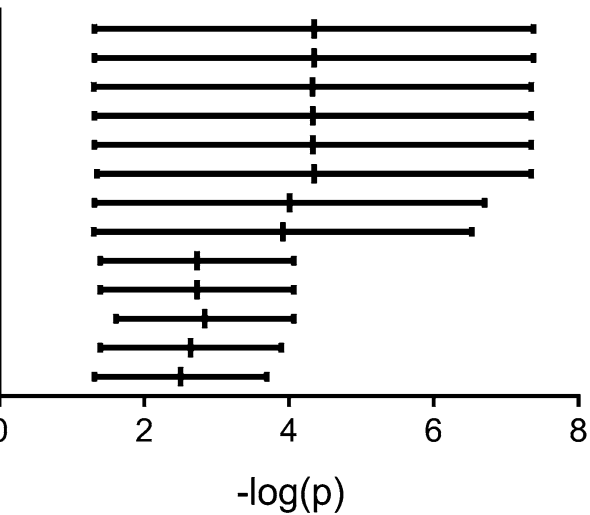

(b)

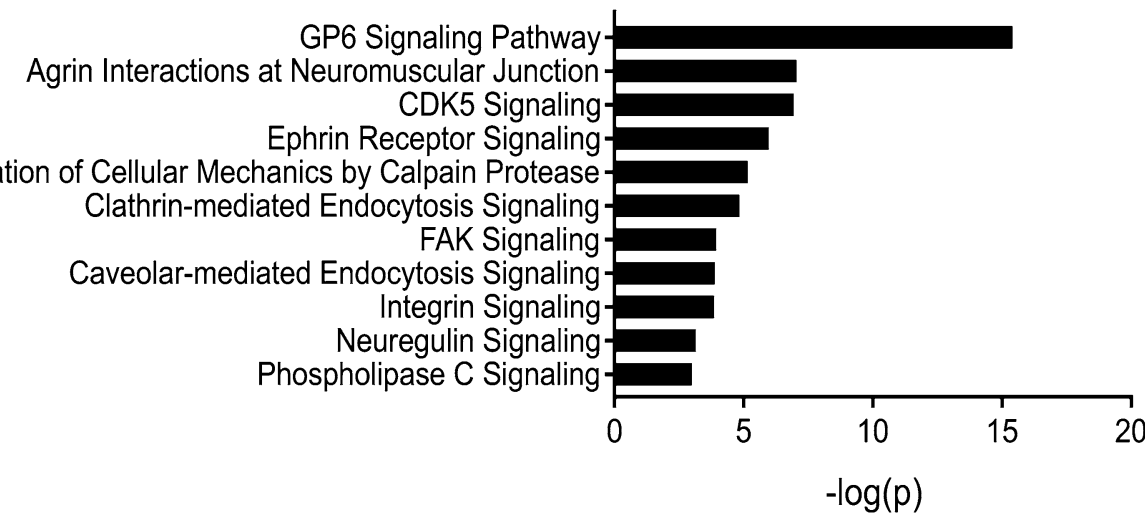


Table 5 IPA gene list for proteomic data (physiological pathways: IPA was also performed using proteomic data and assigned to physiological)

\begin{tabular}{|c|c|c|}
\hline Function & $-\log _{10} \mathrm{p}$ value & Protein \\
\hline Cellular assembly and organization & $1.32-7.38$ & $\begin{array}{l}\text { FLOT1,VCL,GPC1,GPM6A,LAMB1,LAMC1,ADAM10,A2M,TNR,FN1,ITGB1 } \\
\text {,TNC,BCAN,SDCBP,LAMA4,LAMB2,RAP2A,PTPRZ1,SRC,LAMA1,SDC2, } \\
\text { ATP,LTF,CNTN1,CLU,FAT4,KIDINS220,AGRN,NCAN,RAP1B,HSPG2,APO } \\
\text { E,TENM4 }\end{array}$ \\
\hline Cellular function and maintenance & $1.32-7.38$ & $\begin{array}{l}\text { FLOT1,VCL,SYT1,GPC1,GPM6A,LAMB1,LAMC1,ADAM10,A2M,TNR,FN1,I } \\
\text { TGB1,TNC,BCAN,SDCBP,LAMB2,RAP2A,PTPRZ1,SRC,LAMA1,SDC2,MF } \\
\text { GE8,ATP,LTF,CNTN1,CLU,KIDINS220,AGRN,NCAN,RAP1B,APOE,TENM4 }\end{array}$ \\
\hline Cell morphology & $1.35-7.35$ & $\begin{array}{l}\text { FLOT1,VCL,GPM6A,LAMB1,ITGA2,LAMC1,ADAM10,A2M,TNR,FN1,ITGB1 } \\
\text {,TNC,SLC1A3,LAMA4,LAMB2,CD81,RAP2A,PTPRZ1,SRC,LAMA1,GPM6B } \\
\text {,SDC2,CHMP4B,MFGE8,ATP,LTF,CNTN1,CLU,VCAN,KIDINS220,RAP1B,A } \\
\text { GRN,HSPG2,APOE,TENM4 }\end{array}$ \\
\hline Cellular development & $1.32-7.35$ & $\begin{array}{l}\text { FLOT1,TF,VCL,GPC1,GPM6A,LAMB1,LAMC1,ADAM10,A2M,TNR,FN1,ITG } \\
\text { B1,TNC,BCAN,SLC1A3,SDCBP,LAMA4,LAMB2,RAP2A,PTPRZ1,SRC,LA } \\
\text { MA1,SDC2,ATP,CNTN1,CLU,KIDINS220,VCAN,HBA1/HBA2,AGRN,NCAN } \\
\text {,RAP1B,APOE,GPC2,TENM4,NEDD4L }\end{array}$ \\
\hline Cellular growth and proliferation & $1.32-7.35$ & $\begin{array}{l}\text { FLOT1,TF,VCL,GPC1,GPM6A,LAMB1,LAMC1,ADAM10,A2M,TNR,FN1,ITG } \\
\text { B1,TNC,BCAN,SLC1A3,SDCBP,LAMA4,LAMB2,RAP2A,PTPRZ1,SRC,LA } \\
\text { MA1,SDC2,ATP,CNTN1,CLU,KIDINS220,VCAN,HBA1/HBA2,AGRN,NCAN } \\
\text {,RAP1B,APOE,TENM4,NEDD4L }\end{array}$ \\
\hline Nervous system development and function & $1.31-7.35$ & $\begin{array}{l}\text { VCL,LAMB1,LAMC1,TNR,TNC,SDCBP,RAP2A,CD81,PTPRZ1,SRC,LAMA1 } \\
\text {,GPM6B,CHMP4B,LTF,CLU,VCAN,HBA1/HBA2,AGRN,RAP1B,NCAN,HSP } \\
\text { G2,GPC2,NEDD4L,FLOT1,TF,SYT1,GPC1,GPM6A,ITGA2,ATRN,ADAM10, } \\
\text { LAMA5,A2M,FN1,ITGB1,BCAN,SLC1A3,LAMA4,CXADR,LAMB2,SDC2,C } \\
\text { OL3A1,MFGE8,ATP,COL18A1,CNTN1,FAT4,KIDINS220,COL2A1,APOE,T } \\
\text { ENM4,PDCD6IP }\end{array}$ \\
\hline Neurological disease & $1.32-6.71$ & $\begin{array}{l}\text { ANXA7,TF,VCL,LAMB1,LAMC1,RBP4,COL1A1,KRT14,A2M,TNR,C4A/C4B, } \\
\text { FN1,ITGB1,AOX1,BCAN,SLC1A3,LAMA4,LAMB2,CD81,PTPRZ1,SRC,CFI, } \\
\text { COL3A1,GPM6B,CHMP4B,H3F3A/H3F3B,ANXA2,MFGE8,ATP,COL18A1,L } \\
\text { TF,CNTN1,CLU,COL11A1,VCAN,KIDINS220,AGRN,LPL,RAP1B,HSPG2,C } \\
\text { OL2A1,APOE,HPX,PDCD6IP }\end{array}$ \\
\hline Cell-to-cell signalling and interaction & $1.31-6.53$ & $\begin{array}{l}\text { FLOT1,SRC,SYT1,GPM6A,LAMC1,MFGE8,ATP,ADAM10,TNR,CNTN1,FN1, } \\
\text { ITGB1,KIDINS220,AGRN,TNC,NCAN,HSPG2,BCAN,APOE,SDCBP,SLC1A } \\
\text { 3,LAMB2 }\end{array}$ \\
\hline Amino acid metabolism & $1.61-4.07$ & TNR,SRC,SLC1A3,ATP \\
\hline Molecular transport & $1.39-4.07$ & A2M,TNR,SRC,SYT1,ANXA2,APOE,ATP,SLC1A3 \\
\hline Small molecule biochemistry & $1.39-4.07$ & A2M,TNR,SRC,ITGB1,ATP,SLC1A3,APOE \\
\hline Cellular movement & $1.39-3.90$ & $\begin{array}{l}\text { PTPRZ1,SRC,LAMA1,SYT1,GPM6A,COL3A1,LAMC1,ADAM10,A2M,TNR,F } \\
\text { N1,ITGB1,TNC,SLC1A3 }\end{array}$ \\
\hline Cell death and survival & $1.31-3.70$ & $\begin{array}{l}\text { PTPRZ1,SRC,TF,GPC1,GPM6B,ITGA2,LAMC1,ATP,XPR1,A2M,FN1,CLU,ITG } \\
\text { B1,KIDINS220,AGRN,APOE,SLC1A3,LAMA4,PDCD6IP }\end{array}$ \\
\hline
\end{tabular}


Table 6 IPA gene list for proteomic data (canonical pathways)

\begin{tabular}{|c|c|c|}
\hline Pathway & $-\log _{10} \mathrm{p}$ value & Protein \\
\hline GP6 signalling pathway & 15.4 & $\begin{array}{l}\text { COL6A3,LAMA1,COL3A1,LAMB1,LAMC1,COL1A2,COL1A1,ADA } \\
\text { M10,COL18A1,LAMA5,COL5A1,COL11A1,RAP1B,COL2A1,LAM } \\
\text { A4,LAMB2 }\end{array}$ \\
\hline Agrin interactions at neuromuscular junction & 7.05 & SRC,ITGB1,LAMB1,ITGA2,AGRN,RAP1B,LAMC1,RAP2A \\
\hline CDK5 signalling & 6.94 & LAMA1,ITGB1,LAMB1,ITGA2,RAP1B,LAMC1,ATP,LAMA5,RAP2A \\
\hline Ephrin receptor signalling & 5.97 & $\begin{array}{l}\text { MAP4K4,SRC,ITGB1,ITGA2,SDC2,RAP1B,GNB1,SDCBP,ADAM10 } \\
\text { „RAP2A }\end{array}$ \\
\hline Regulation of cellular mechanics by calpain protease & 5.16 & SRC,VCL,ITGB1,ITGA2,RAP1B,RAP2A \\
\hline Clathrin-mediated endocytosis signalling & 4.84 & TSG101,RAB5C,RAB7A,TF,SRC,CLU,ITGB1,RBP4,APOE \\
\hline FAK signalling & 3.94 & SRC,VCL,ITGB1,ITGA2,RAP1B,RAP2A \\
\hline Caveolar-mediated endocytosis signalling & 3.88 & FLOT1,RAB5C,SRC,ITGB1,ITGA2 \\
\hline Integrin signalling & 3.86 & TSPAN6,SRC,VCL,ITGB1,ITGA2,RAP1B,TSPAN7,RAP2A \\
\hline Neuregulin signalling & 3.15 & SRC,ITGB1,ITGA2,RAP1B,RAP2A \\
\hline Phospholipase $\mathrm{C}$ signalling & 2.99 & SRC,ITGB1,PLD3,ITGA2,RAP1B,GNB1,RAP2A \\
\hline
\end{tabular}

Acknowledgements The authors thank the core facilities in the Faculty of Biology, Medicine and Health at the University of Manchester, specifically: the staff in the Electron Microscopy Core Facility, especially Dr. Aleksandr Mironov, for their assistance, and the Wellcome Trust for equipment grant support; Dr. Tom Jowitt in the Biomolecular Analysis Core Facility; Dr. Rachel Scholey and Dr. Andy Hayes of the Bioinformatics and Genomic Technologies Core Facilities for providing support with RNA-seq; Dr. David Knight and Dr. Julian Selley in the Biological Mass Spectrometry Facility for support with proteomic analysis.

Author Contributions DH, SPB and NH designed the study; DH, SPB, $\mathrm{NH}$ and MA acquired funding; $\mathrm{DH}, \mathrm{NC}, \mathrm{KF}$ and $\mathrm{AJ}$ performed experiments; DH and AJ analysed data; $\mathrm{DH}$ and $\mathrm{NH}$ wrote the manuscript; $\mathrm{DH}, \mathrm{NH}$ and MA edited the manuscript.

Funding DH is supported by an Alzheimer's Society fellowship. Additional funding was provided by an Alzheimer's Research UK Small Network Grant to DH and MA. iPSC culture was funded by grants from the Medical Research Council (MRC) (MR/M024997/1 and MR/ N013255/1), and the Donald Dean Fund for Dementia Research to NH.

\section{Compliance with Ethical Standards}

Conflict of interest The authors declare that the research was conducted in the absence of any commercial or financial relationships that could be construed as a potential conflict of interest.

Open Access This article is licensed under a Creative Commons Attribution 4.0 International License, which permits use, sharing, adaptation, distribution and reproduction in any medium or format, as long as you give appropriate credit to the original author(s) and the source, provide a link to the Creative Commons licence, and indicate if changes were made. The images or other third party material in this article are included in the article's Creative Commons licence, unless indicated otherwise in a credit line to the material. If material is not included in the article's Creative Commons licence and your intended use is not permitted by statutory regulation or exceeds the permitted use, you will need to obtain permission directly from the copyright holder. To view a copy of this licence, visit http://creativecommons.org/licenses/by/4.0/.

\section{References}

1. Caruso Bavisotto C, Scalia F, Marino Gammazza A, Carlisi D, Bucchieri F, Conway de Macario E et al (2019) Extracellular vesicle-mediated cell-cell communication in the nervous system: focus on neurological diseases. Int J Mol Sci. 20(2):E434

2. van Niel G, D'Angelo G, Raposo G (2018) Shedding light on the cell biology of extracellular vesicles. Nat Rev Mol Cell Biol 19(4):213-228

3. Colombo M, Raposo G, Thery C (2014) Biogenesis, secretion, and intercellular interactions of exosomes and other extracellular vesicles. Annu Rev Cell Dev Biol 30:255-289

4. Johnstone RM, Adam M, Hammond JR, Orr L, Turbide C (1987) Vesicle formation during reticulocyte maturation. Association of plasma membrane activities with released vesicles (exosomes). J Biol Chem 262(19):9412-9420

5. Beit-Yannai E, Tabak S, Stamer WD (2018) Physical exosome:exosome interactions. J Cell Mol Med 22(3):2001-2006

6. Morelli AE, Larregina AT, Shufesky WJ, Sullivan ML, Stolz DB, Papworth GD et al (2004) Endocytosis, intracellular sorting, and processing of exosomes by dendritic cells. Blood 104(10):3257-3266

7. Valadi H, Ekstrom K, Bossios A, Sjostrand M, Lee JJ, Lotvall JO (2007) Exosome-mediated transfer of mRNAs and microRNAs is a novel mechanism of genetic exchange between cells. Nat Cell Biol 9(6):654-659

8. Pastuzyn ED, Day CE, Kearns RB, Kyrke-Smith M, Taibi AV, McCormick J et al (2018) The neuronal gene arc encodes a repurposed retrotransposon gag protein that mediates intercellular RNA transfer. Cell 172(1-2):275-288

9. Quek C, Hill AF (2017) The role of extracellular vesicles in neurodegenerative diseases. Biochem Biophys Res Commun 483(4):1178-1186

10. Kucharzewska P, Christianson HC, Welch JE, Svensson KJ, Fredlund E, Ringner M et al (2013) Exosomes reflect the hypoxic status of glioma cells and mediate hypoxia-dependent activation of vascular cells during tumor development. Proc Natl Acad Sci USA 110(18):7312-7317

11. Ludwig N, Whiteside TL, Reichert TE (2019) Challenges in exosome isolation and analysis in health and disease. Int J Mol Sci. 20(19):4684 
12. Meng W, Hao Y, He C, Li L, Zhu G (2019) Exosome-orchestrated hypoxic tumor microenvironment. Mol Cancer 18(1):57

13. Meng Y, Eirin A, Zhu XY, O'Brien DR, Lerman A, van Wijnen AJ et al (2018) The metabolic syndrome modifies the mRNA expression profile of extracellular vesicles derived from porcine mesenchymal stem cells. Diabetol Metab Syndr 10:58

14. King HW, Michael MZ, Gleadle JM (2012) Hypoxic enhancement of exosome release by breast cancer cells. BMC Cancer 12:421

15. de Jong OG, Verhaar MC, Chen Y, Vader P, Gremmels H, Posthuma $\mathrm{G}$ et al (2012) Cellular stress conditions are reflected in the protein and RNA content of endothelial cell-derived exosomes. J Extracell Vesicles 1:18396.

16. Hurley JH (2008) ESCRT complexes and the biogenesis of multivesicular bodies. Curr Opin Cell Biol 20(1):4-11

17. Thery C, Amigorena S, Raposo G, Clayton A (2006) Isolation and characterization of exosomes from cell culture supernatants and biological fluids. Curr Protoc Cell Biol 30:3-22

18. Yasukawa M, Hato T, Inatsuki A, Kobayashi Y (1988) Expression of CD9 (p24) antigen on hematopoietic cells following treatment with phorbol ester. Acta Haematol 79(3):133-136

19. Ostrowski M, Carmo NB, Krumeich S, Fanget I, Raposo G, Savina A et al (2010) Rab27a and Rab27b control different steps of the exosome secretion pathway. Nat Cell Biol 12(1):19-30 (sup pp 1-13)

20. Guo BB, Bellingham SA, Hill AF (2015) The neutral sphingomyelinase pathway regulates packaging of the prion protein into exosomes. J Biol Chem 290(6):3455-3467

21. Trajkovic K, Hsu C, Chiantia S, Rajendran L, Wenzel D, Wieland $F$ et al (2008) Ceramide triggers budding of exosome vesicles into multivesicular endosomes. Science 319(5867):1244-1247

22. Jahn R, Scheller RH (2006) SNAREs-engines for membrane fusion. Nat Rev Mol Cell Biol 7(9):631-643

23. Zagrean AM, Hermann DM, Opris I, Zagrean L, Popa-Wagner A (2018) Multicellular crosstalk between exosomes and the neurovascular unit after cerebral ischemia. Ther Imp Front Neurosci $12: 811$

24. van Wilgenburg B, Browne C, Vowles J, Cowley SA (2013) Efficient, long term production of monocyte-derived macrophages from human pluripotent stem cells under partly-defined and fullydefined conditions. PLoS ONE 8(8):e71098

25. Moxon SR, Corbett NJ, Fisher K, Potjewyd G, Domingos M, Hooper NM (2019) Blended alginate/collagen hydrogels promote neurogenesis and neuronal maturation. Mater Sci Eng C 104:109904

26. Jarosz-Griffiths HH, Corbett NJ, Rowland HA, Fisher K, Jones AC, Baron J et al (2019) Proteolytic shedding of the prion protein via activation of metallopeptidase ADAM10 reduces cellular binding and toxicity of amyloid-beta oligomers. J Biol Chem 294(17):7085-7097

27. Shi Y, Kirwan P, Livesey FJ (2012) Directed differentiation of human pluripotent stem cells to cerebral cortex neurons and neural networks. Nat Protoc 7(10):1836-1846

28. Grillo G, Turi A, Licciulli F, Mignone F, Liuni S, Banfi S et al (2010) UTRdb and UTRsite (RELEASE 2010): a collection of sequences and regulatory motifs of the untranslated regions of eukaryotic mRNAs. Nucleic Acids Res. 38:D75-80

29. Wang D (2018) GCevobase: an evolution-based database for GC content in eukaryotic genomes. Bioinformatics 34(12):2129-2131

30. Dhapola P, Chowdhury S (2016) QuadBase2: web server for multiplexed guanine quadruplex mining and visualization. Nucleic Acids Res 44(W1):W277-W283

31. Chang H, Lim J, Ha M, Kim VN (2014) TAIL-seq: genome-wide determination of poly(A) tail length and 3' end modifications. Mol Cell 53(6):1044-1052

32. Li M, Zeringer E, Barta T, Schageman J, Cheng A, Vlassov AV (2014) Analysis of the RNA content of the exosomes derived from blood serum and urine and its potential as biomarkers. Philos Trans R Soc Lond B. 369(1652):20130502

33. Yuan T, Huang X, Woodcock M, Du M, Dittmar R, Wang Y et al (2016) Plasma extracellular RNA profiles in healthy and cancer patients. Sci Rep 6:19413

34. Li J, Chen X, Yi J, Liu Y, Li D, Wang J et al (2016) Identification and characterization of $293 \mathrm{~T}$ cell-derived exosomes by profiling the protein, mRNA and MicroRNA components. PLoS ONE 11(9):e0163043

35. Huang X, Yuan T, Tschannen M, Sun Z, Jacob H, Du M et al (2013) Characterization of human plasma-derived exosomal RNAs by deep sequencing. BMC Genomics 14:319

36. Takahashi A, Okada R, Nagao K, Kawamata Y, Hanyu A, Yoshimoto $\mathrm{S}$ et al (2017) Exosomes maintain cellular homeostasis by excreting harmful DNA from cells. Nat Commun 8:15287

37. Maia J, Caja S, Strano Moraes MC, Couto N, Costa-Silva B (2018) Exosome-based cell-cell communication in the tumor microenvironment. Front Cell Dev Biol 6:18

38. Shabbir A, Cox A, Rodriguez-Menocal L, Salgado M, Van Badiavas E (2015) Mesenchymal stem cell exosomes induce proliferation and migration of normal and chronic wound fibroblasts, and enhance angiogenesis in vitro. Stem Cells Dev 24(14):1635-1647

39. Qu JL, Qu XJ, Zhao MF, Teng YE, Zhang Y, Hou KZ et al (2009) Gastric cancer exosomes promote tumour cell proliferation through PI3K/Akt and MAPK/ERK activation. Dig Liver Dis 41(12):875-880

40. Hock A, Miyake H, Li B, Lee C, Ermini L, Koike Y et al (2017) Breast milk-derived exosomes promote intestinal epithelial cell growth. J Pediatr Surg 52(5):755-759

41. Paolillo M, Schinelli S (2017) Integrins and exosomes, a dangerous liaison in cancer progression. Cancers (Basel). 9(8):95

42. Tian T, Zhu YL, Zhou YY, Liang GF, Wang YY, Hu FH et al (2014) Exosome uptake through clathrin-mediated endocytosis and macropinocytosis and mediating miR-21 delivery. J Biol Chem 289(32):22258-22267

43. Mulcahy LA, Pink RC, Carter DR (2014) Routes and mechanisms of extracellular vesicle uptake. J Extracell Vesicles 3:24641

44. McKelvey KJ, Powell KL, Ashton AW, Morris JM, McCracken SA (2015) Exosomes: mechanisms of uptake. J Circ Biomark $4: 7$

45. Mirzapoiazova T, Lennon FE, Mambetsariev B, Allen M, Riehm J, Poroyko VA et al (2015) Extracellular vesicles from caveolinenriched microdomains regulate hyaluronan-mediated sustained vascular integrity. Int J Cell Biol 2015:481493

46. Clayton A, Turkes A, Dewitt S, Steadman R, Mason MD, Hallett MB (2004) Adhesion and signaling by B cell-derived exosomes: the role of integrins. FASEB J 18(9):977-979

47. Gautam M, Noakes PG, Moscoso L, Rupp F, Scheller RH, Merlie JP et al (1996) Defective neuromuscular synaptogenesis in agrindeficient mutant mice. Cell 85(4):525-535

48. An M, Zhu J, Wu J, Cuneo KC, Lubman DM (2018) Circulating microvesicles from pancreatic cancer accelerate the migration and proliferation of PANC-1 cells. J Proteom Res 17(4):1690-1699

49. Zhao Y, Yin L, Zhang H, Lan T, Li S, Ma P (2018) Eph/ephrin family anchored on exosome facilitate communications between cells. Cell Biol Int 42(11):1458-1462

50. Yue Y, Wang C, Benedict C, Huang G, Truongcao M, Roy R et al (2020) Interleukin-10 deficiency alters endothelial progenitor cell-derived exosome reparative effect on myocardial repair via integrin-linked kinase enrichment. Circ Res 126(3):315-329

51. Sun B, Ma Y, Wang F, Hu L, Sun Y (2019) miR-644-5p carried by bone mesenchymal stem cell-derived exosomes targets regulation of p53 to inhibit ovarian granulosa cell apoptosis. Stem Cell Res Ther 10(1):360 
52. Yamashita T, Kamada H, Kanasaki S, Nagano K, Inoue M, Higashisaka K et al (2019) Ephrin type-A receptor 2 on tumorderived exosomes enhances angiogenesis through the activation of MAPK signaling. Pharmazie 74(10):614-619

53. Sheldon H, Heikamp E, Turley H, Dragovic R, Thomas P, Oon CE et al (2010) New mechanism for Notch signaling to endothelium at a distance by Delta-like 4 incorporation into exosomes. Blood 116(13):2385-2394

54. Gross JC, Chaudhary V, Bartscherer K, Boutros M (2012) Active Wnt proteins are secreted on exosomes. Nat Cell Biol 14(10):1036-1045

55. de Boni L, Wullner U (2019) Epigenetic analysis in human neurons: considerations for disease modeling in PD. Front Neurosci 13:276

56. Potjewyd G, Moxon S, Wang T, Domingos M, Hooper NM (2018) Tissue engineering 3D neurovascular units: a biomaterials and bioprinting perspective. Trends Biotechnol 36(4):457-472

57. van der Vos KE, Abels ER, Zhang X, Lai C, Carrizosa E, Oakley D et al (2016) Directly visualized glioblastoma-derived extracellular vesicles transfer RNA to microglia/macrophages in the brain. Neuro-Oncol 18(1):58-69
58. Waldenstrom A, Genneback N, Hellman U, Ronquist G (2012) Cardiomyocyte microvesicles contain DNA/RNA and convey biological messages to target cells. PLoS ONE 7(4):e34653

59. Ekstrom K, Valadi H, Sjostrand M, Malmhall C, Bossios A, Eldh $\mathrm{M}$ et al (2012) Characterization of mRNA and microRNA in human mast cell-derived exosomes and their transfer to other mast cells and blood CD34 progenitor cells. J Extracell Vesicles. 1:18389

60. Ramachandran S, Palanisamy V (2012) Horizontal transfer of RNAs: exosomes as mediators of intercellular communication. Wiley Interdiscip Rev RNA 3(2):286-293

Publisher's Note Springer Nature remains neutral with regard to jurisdictional claims in published maps and institutional affiliations. 